\title{
Wireless Communication for Gas Detection using 433RF Modules and Arduino Processor
}

\author{
Zahraa K. Taha \\ Asst. Lecture, Department of Network Engineering, College of Engineering, \\ Al-Iraqia University, Baghdad, Iraq.
}

\begin{abstract}
$\mathrm{RF}$ is any of the electromagnetic wave frequencies that lie in the range extending from around $3 \mathrm{kHz}$ to $300 \mathrm{GHz}$, which include those frequencies used for communications or radar signals. These RF modules are very popular among the Arduino tinkerers. The $433 \mathrm{MHz}$ is used on a wide variety of applications that require wireless control. These modules are very cheap and you can use them with any microcontroller. In this paper, Gas sensor is connected to the $433 \mathrm{RF}$ modules. The Gas sensor outputs a voltage that is proportional to the concentration of smoke/gas. If the sensor detects the gas, the $\mathrm{RF}$ modules send alarm message
\end{abstract}

\section{Keywords}

Arduino Uno, RF Module, MQ-2 gas sensor.

\section{INTRODUCTION}

Arduino is an open source single board-based microcontroller. It is a very popular platform forked from the Wiring platform and firstly designed to popularize the use of electronics in interaction design university students' projects. It is based on the Atmel AVR processor and provides many inputs and outputs in only one self-sufficient piece of hardware. The project was started in Italy in 2005 by founders Massimo Banzi and David Cuartielles. Today it is one of the most beautiful examples of the open source concept, brought to the hardware world and being often used only in the software world [1].

Wireless communication is among technology's biggest contributions to mankind. Wireless communication involves the transmission of information over a distance without help of wires, cables or any other forms of electrical conductors. The transmitted distance can be anywhere between a few meters (for example, a television's remote control) and thousands of kilometres (for example, radio communication) [2]. In this paper text information is send in one direction using a wireless link between two Arduino-controlled systems that have inexpensive radio frequency (433RF) data module [3].

\section{RF (TRANSMITTER \& RECEIVER) MODULE}

The RF module, as the name suggests, operates at Radio Frequency. The corresponding frequency range varies between $30 \mathrm{kHz} \& 300 \mathrm{GHz}$. In this RF system, the digital data is represented as variations in the amplitude of carrier wave. This kind of modulation is known as Amplitude Shift Keying (ASK). Transmission through RF is better than IR (infrared) because of many reasons. Firstly, signals through RF can travel through larger distances making it suitable for long range applications. Also, while IR mostly operates in line-of-sight mode, RF signals can travel even when there is an obstruction between transmitter \& receiver. Next, RF transmission is more strong and reliable than IR transmission.
RF communication uses a specific frequency unlike IR signals which are affected by other IR emitting sources. This RF module comprises of an RF Transmitter and an RF Receiver. The transmitter/receiver $(\mathrm{Tx} / \mathrm{Rx})$ pair operates at a frequency of $434 \mathrm{MHz}$. An RF transmitter receives serial data and transmits it wirelessly through RF through its antenna connected at pin4. The transmission occurs at the rate of $1 \mathrm{Kbps}-10 \mathrm{Kbps}$. The transmitted data is received by an RF receiver operating at the same frequency as that of the transmitter. The RF module is often used along with a pair of encoder/decoder. The encoder is used for encoding parallel data for transmission feed while reception is decoded by a decoder. HT12E-HT12D, HT640-HT648, etc. are some commonly used encoder/decoder pair ICs [4].

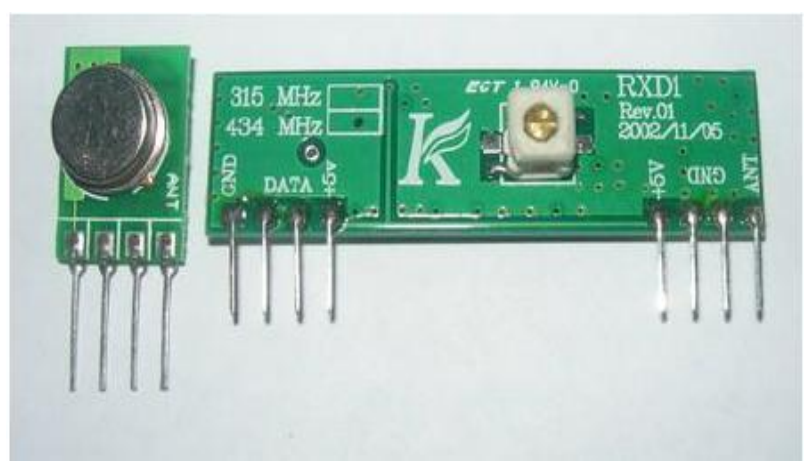

Fig 1: RF Module

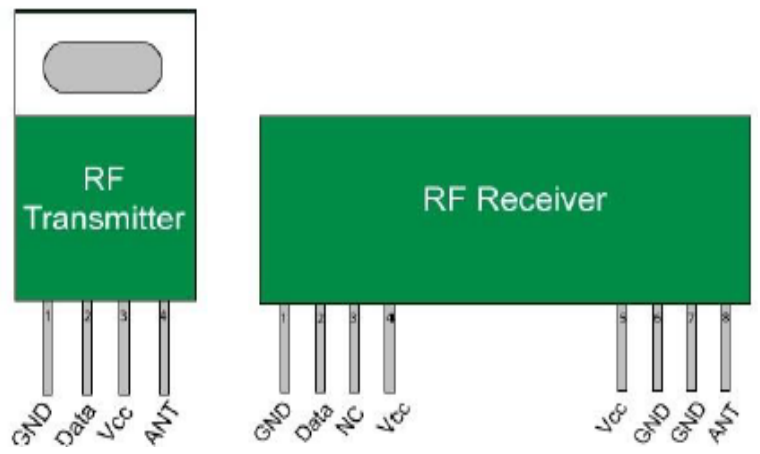

Fig 2: PIN diagram of RF Module

Basic Specification: [5]

- Frequency: 433Mhz.

- Modulation: ASK

- Receiver data output: High - 1/2 Vcc, Low - 0.7v

- Transmitor input voltage: $3-12 \mathrm{~V}$ (high voltage $=$ more transmitting power)

- Transmitting range (work at $5 \mathrm{~V}$ ): $40 \mathrm{~m}$ indoor, and $100 \mathrm{~m}$ in open air 


\section{MQ-2 GAS SENSOR}

The MQ-2 smoke sensor is sensitive to smoke and to the following flammable gases: LPG, Butane ...etc. The smoke sensor has a built-in potentiometer that allows you to adjust the sensor sensitivity according to how accurate you want to detect gas.The voltage that the sensor outputs changes accordingly to the smoke/gas level that exists in the atmosphere. The sensor outputs a voltage that is proportional to the concentration of smoke/gas [6].

\section{PROJECT}

In this paper, alarm system is made to detect anybody that would walk through the house. It consist from two parts: an emitter part with the Gas sensor, and a receiver part that receiver part that will be the "hub" of the project. The materials are needed:

Two Arduino UNO

$433 \mathrm{RF}$ transmitting-receiver modules.

Gas sensor

Jumper wires

Transmitter:

For the transmitter that is connected to the sensor. First, connect the transmitter. It also has three pins to connect: VCC, GND, and data. The MQ-2 sensor has 4 pins (VCC, GND, A0 and D0). D0 is connected to any digital pins.

\#include <VirtualWire.h>

const int led_pin $=13$;

const int transmit_pin $=10$;

const int sensor_pin $=4$;

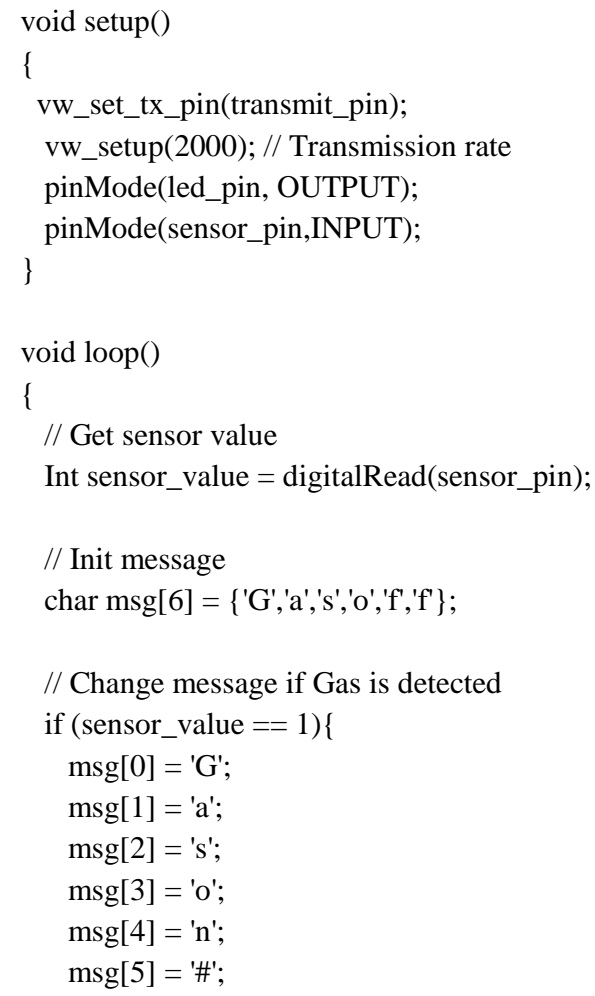

// Transmit data every second digitalWrite(led_pin, HIGH); // Flash a light to show transmitting vw_send((uint8_t *)msg, 6); vw_wait_tx(); // Wait until the whole message is gone digitalWrite(led_pin, LOW); delay(1000);

Reciever:

\#include <VirtualWire.h>

// Pins definition

const int led_pin $=13$;

const int transmit_pin $=10$;

const int receive_pin $=11$;

void $\operatorname{setup}()$

\{

// Init

delay(1000);

Serial.begin(9600); // Debugging only

Serial.println("setup");

// Initialise the IO and ISR

vw_set_rx_pin(receive_pin);

vw_setup(2000); // Transmission rate

// Start the receiver PLL

vw_rx_start();

// Set LED pin

pinMode(led_pin, OUTPUT);

\}

void $\operatorname{loop}()$

\{

uint8_t buf[VW_MAX_MESSAGE_LEN];

uint8_t buflen $=$ VW_MAX_MESSAGE_LEN;

// Check if a message was received

if (vw_get_message(buf, \&buflen))

\{

// Flash a light to show received good message

digitalWrite(led_pin, HIGH);

Serial.print("Got: ");

// Print message

for (int $\mathrm{i}=0$; $\mathrm{i}<$ buflen; $\mathrm{i}++$ )

\{

Serial.print(char(buf[i]));

Serial.print(' ');

\}

Serial.println();

digitalWrite(led_pin, LOW);

\}

\} 


\section{CONCLUSION}

These 433 RF transmitter/receiver modules are pretty simple and cheap serial devices. The transmitter sends serial data to the receiver through a simplex transmission method. It often used as garage door opener. Useful for sensor network can operate with this module.

The RF Link Modules are used for Radio frequency transmission. Normally when we think about wireless communication to be used on Arduino, the first thing that comes to mind for us is the famous Xbee. However not everyone has the option to buy an Xbee, due to it being relatively expensive compared to the RF Link Module. It is easy to use rather than wireless communication methods.

\section{REFERENCES}

[1] J. Bayle "C Programming for Arduino" May 2013.
[2] Wireless Communication and types, By: Rose Mary https://www.engineersgarage.com/articles/wireless_com munication.

[3] J. Boxball "Arduino Workshop: A Hands-On Introduction with 65 Projects" 1st edition, 2013 (AJER), Vol-5, Issue-10, pp-358-362

[4] F. Ahmed, S. Alim, S. Islam, K. Bhusan and S. Islam "433 MHz (Wireless RF) Communication between Two Arduino UNO", American Journal of Engineering Research (AJER), Vol-5, Issue-10, pp-358-362

[5] 433Mhz $\mathrm{RF}$ link kit https://www.seeedstudio.com/433mhz-rf-link-kit-p127.html

[6] Smoke Detection using MQ-2 Gas Sensor https://create.arduino.cc/projecthub/Aritro/smokedetection-using-mq-2-gas-sensor-79c54a 